\title{
Lung involvement of an infectious disease having increasing incidence
}

\author{
Lale Sertçelik $^{1}$, Tulin Kuyucu ${ }^{1}$, Fatma Armagan Hazar ${ }^{1}$, Gulgun Cetintas Afsar ${ }^{1 *}$ and Semra Bati Kutlu ${ }^{2}$ \\ ${ }^{1}$ University of Heath Sciences Sureyyapasa Chest Disease and Chest Surgery Training and Research Hospital, Chest Diseases, Turkey \\ ${ }^{2}$ University of Heath Sciences Sureyyapasa Chest Disease and Chest Surgery Training and Research Hospital, infection diseases, Turkey
}

\begin{abstract}
Backgroud: The incidence of HIV/AIDS infection is gradually increasing. One of the organs, which this infection targets, is the lungs. The lung involvement is seen in forms of recurrent pneumonia, pneumocystis jiroveci pneumonia, tuberculosis, and Kaposi sarcoma.

Aim: The aim of this study is to draw attention on the importance of this subject while examining the cases, which we follow up in hospital.

Study design: The data were obtained from the files and hospital records of all of HIV (+) patients, who have been hospitalized in our hospital in last 10 years.

Methods: a retrospective study

Results: On the contrary with literature, most of our HIV (+) patients were diagnosed for P.jiroveci pneumonia. For the patients applying with complaints of cough, fever, and dyspnea and then found to have deep hypoxia and extensive infiltration in lung and oral candidiasis in physical examination, P.jiroveci pneumonia should be considered, thoracic CT - HRCT should be immediately taken and HIV test should be performed. Starting the accurate therapy as soon as possible significantly affects the clinical course. For the patients diagnosed for tuberculosis, the HIV serology should be requested, and consequently the antiretroviral treatment should be initiated according to the results.

Conclusions: According to our study, $83 \%$ of the positivity of HIV was determined through the respiratory system complications of this infectious disease. It was aimed to draw attention to this infectious disease having increasing incidence, and to raise awareness that performing the required laboratory tests affects the prognosis.

Moreover, 4 patients known to be HIV (+) gave no information on this subject in anamnesis. According to the practice of Ministry of Health, HIV serology shall not be performed unless the approval of patient is obtained. But, this also poses risk for the healthcare professionals. We believe that the required legal regulations taking healthcare professionals under protection should be prepared.
\end{abstract}

\section{Introduction}

HIV/AIDS disease was defined for the first time in USA in year 1981 through rarely seen Pneumocystis jirowici (older name Pneumocystis carini) pneumonia and Kaposi sarcoma (KS) [1]. With 2.1 million newly diagnosed for HIV infection in year 2015, there are totally 36.7 million people living with HIV, but only 17 million of them receive anti-retroviral treatment. It was reported that 25.3 million individuals have lost their lives because of this disease since year 2000 [2].

First HIV/AIDS cases (3 cases) were reported in year 1985 in our country, and the number of cases gradually increased year by year. TR Ministry of Health reported that there were 10,475 HIV/AIDS patients in our country in year 2015, but the real number is estimated to be much higher.

One of the main organs affected by HIV is the lungs. In $70 \%$ of the patients, minimum one complication is seen in respiratory system during the course of disease. The complications in respiratory system are the reasons for morbidity and mortality. Pulmonary involvement of HIV can be listed as opportunistic infections, immune restoration syndrome, lymphoproliferative disorders, AIDS-related malignity, non-specific interstitial pneumonia, medication reactions, HIV-related pulmonary hypertension, bronchiolitis obliterans, emphysema, and bronchiectasis [3-6].
The aim of this study is to draw attention on the importance of this subject while examining the cases, which we follow up in hospital.

\section{Materials and methods}

In this study, among the patients that have been hospitalized in our hospital in last 10 years, the patients found to be HIV (+) and the patients, who declared in anamnesis that they were HIV (\%). The data were retrospectively obtained from the patient files and hospital records. We examined the parameters of age, gender, complaint for applying to the hospital, way of determining HIV positivity, laboratory and radiological findings, and the treatment and therapies chosen by a physician.

\section{Results}

In hospital records, it was determined that there were $54 \mathrm{HIV}(+)$ patients hospitalized in our hospital, age of whom ranges between 24

Correspondence to: Gulgun Cetintas Afsar, Department of Pulmonary Medicine, Istanbul Sureyyapasa Chest Diseases and Chest Surgery Hospital, Maltepe, Istanbul, TR 34844, Turkey, E-mail: gulguncet@hotmail.com

Key words: HIV, pneumocystis P. jiroveci pneumonia, tuberculosis, lung involvement

Received: May 20, 2017; Accepted: June 19, 2017; Published: June 21, 2017 
and 74 years. $49(\% 91.75)$ were male and $5(\% 9,25)$ were female. 42 patients didn't know that they were HIV (+), but 12 patients knew. 8 of the patients stated in anamnesis that they were HIV (+), while 4 of patients have not been informed about that they have HIV infection. Only one of the patients was receiving antiretroviral therapy.

$25(46,2 \%)$ patients were followed up for pneumocystis jirowici pneumonia, 16 patients $(29,6 \%)$ were followed up for tuberculosis, 7 patients $(12,9 \%)$ followed up for COPD, 5 patients $(9,2 \%)$ followed up for CMV pneumonia (Figure 1).

The patients that followed up and treated for pneumocystis jirowici pneumonia (PJP) diagnosis; atypical mycobacteria infection was accompanying PJP in 1 of these patients, and 1 patient was diagnosed for multidrug resistance tuberculosis (MDRTb). PJP diagnosis of 21 patients was made clinic-radiologically, the diagnosis was confirmed via trans-bronchial parenchyma biopsy in 2 patients, 1 patient was diagnosed for $P$. jiroveci in bronchus lavage. 1 patient was diagnosed with lung wedge resection. 4 patients were found to have positivity with CMV PCR and they were considered to have CMV pneumonia. One of them had atypical mycobacteria.

Tuberculosis was detected in 16 patients. 2 of them were atypical tuberculosis infection, 1 MDRTb, 1 tuberculosis lymphadenitis, 1 lung tuberculosis + lymph node tuberculosis + Milier TB association, 1 tuberculosis empyema, and 2 tuberculosis pleurisy.

The most frequent symptoms of patients followed-up with diagnosis of PJP were cough, dyspnea, and fever. In 18 of the patients, hypoxia was detected in arterial blood gas. In complete blood count of the patients, leucopenia was detected in $7(\% 28)$ patients, and lymphopenia in 23(\%92) patients. In 9 of 25 patients diagnosed for PCP, oral candidiasis was observed in 9 patients. Bilateral and widespread infiltration was found in chest radiography of 24 (96\%) of patients, while right hilar infiltration was observed in radiography of $1(4 \%)$ patient. Bilateral and extensive ground-glass appearance in thoracic CT. The patient, who had continuing local infiltration despite the implementation of pneumonia treatment, received and responded to the PCP treatment. LDH increase was observed in biochemical results of 22 patients.

In anamnesis, 3 of 25 patients diagnosed for PCP reported that they were HIV (+). For 1 patient, HIV positivity was detected during the

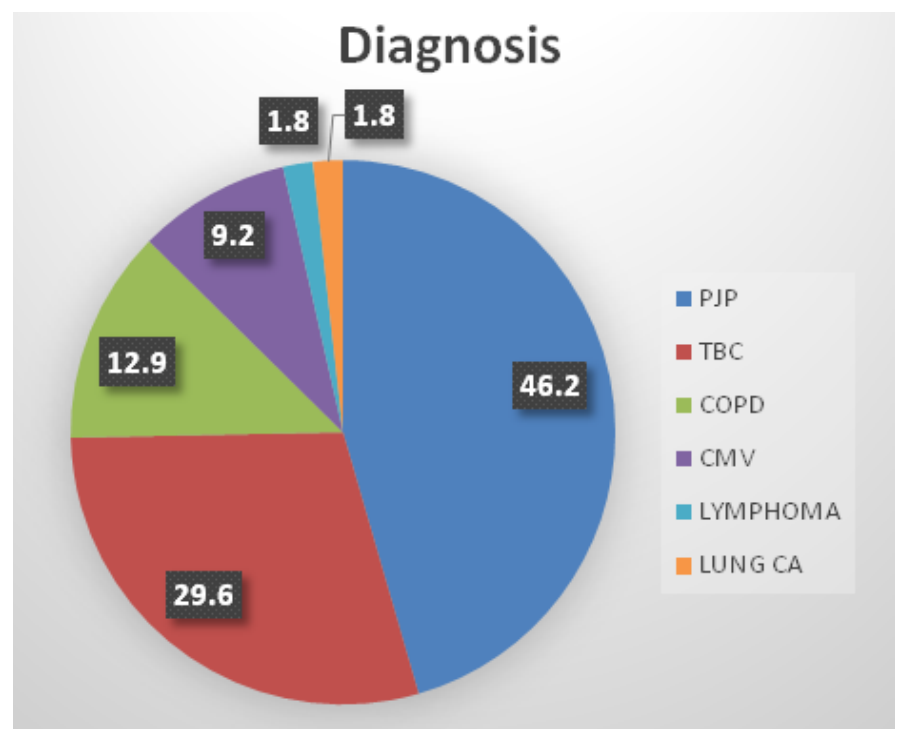

Figure 1. Lung involvement associated with HIV infection preparation for operation based on the pre-diagnosis of interstitial lung disease, while 3 patients didn't mention about their HIV infection even though they knew. After evaluating anamnesis, physical examination, and laboratory and radiology results of 21 patients, HIV positivity was detected via serological examination performed because of the suspicion for PCP. Association of HIV with HCV was observed in 2 patients. In one of the patients, the microbiological factor was shown to be P.jiroveci, while the histopathology consistent with PJP was detected in one patient via trans-bronchial needle aspiration biopsy and in other one via wedge resection performed. For other patients, the treatment was applied based on the clinic-pathological diagnosis of PJP 10 of 25 patients needed intensive care. 3 of them lost their lives in hospital. We believe that the mortality rate would be higher if the pre-diagnosis of $P$.jiroveci was not considered and the appropriate treatment was not started.

In 7 patients followed up with diagnosis of COPD, HIV positivity was detected in our hospital. It was considered as a pre-diagnosis for 5 patients, and HIV serology was requested. It was also requested for application of 1 patient to Poorhouse (Darulaceze) and positivity was detected. It was detected during the preoperative examinations for the patients diagnosed for lung cancer and lymphoma.

A patient, who applied with the complaint of widespread rash and hemoptysis, diagnosed for $P$. Jiroveci pneumonia and Kaposi sarcoma. In Fiber Optic Bronchoscopy (FOB), fragile hemorrhagic mucosa was observed, and the result of bronchus lavage pathology was determined to be non-diagnostic. In cardiac examination, the pulmonary hypertension was found.

While examining the etiology of 2 patients' dyspnea, pulmonary hypertension was found in echocardiography.

\section{Discussion}

One of the target organs of HIV infection is the lungs. The objective in this study is to evaluate the respiratory involvement of HIV (+) patients, who have been hospitalized in Sureyyapasa Chest Diseases and Chest Surgery Training and Research Hospital in last 10 years.

According to a clinical study carried out in USA in year 2000, the most frequently seen respiratory symptom among HIV $(+)$ patients was found to be bacterial infections [7]. Of our patients, $46.2 \%$ were diagnosed for PJP, and $29.62 \%$ for TB. This can be explained with the fact that our hospital is a chest disease hospital. No recurrent pneumonia was observed among our patients. For the patients having recurrent pneumonia, performing no HIV serology examination might be argued as a reason. The most important deficiency regarding this subject is the absence of consensus on this matter.

Among 25 patients diagnosed for PJP, P.jiroveci was found only in 1 patient in BAL and in 2 patients in histopathology. Resting 21 patients were treated with clinic-radiological PJP diagnosis. But, among the patients being followed-up due to HIV positivity, Fiberoptic Bronchoscopy (FOB), and Bronchoalveolar Lavage (BAL) should be routinely applied in early-detection of patient being followed-up due to HIV positivity and in revealing the accompanying pathologies such as Kaposi syndrome, the BAL liquids should be evaluated in terms of bacteria, fungi, and tuberculosis [8]. FOB was applied within 2 weeks to only 2 patients due to their clinic situation and the response to treatment. In order to reveal the factors and accompanying situations, FOB should be implemented. In order to reveal the factors and concomitant conditions, it should be applied to FOB and BAL HIV (+) patients. 
The PA Lung X-Ray images of PJP patients in our study revealed a bilateral interstitial involvement. When the interstitial involvement is seen in PA lung $\mathrm{x}$-ray, it was recommended in literature to have HRCT imaging, as in our study. Moreover, ground-glass appearance was present in HRCT. The radiologic images of our patients diagnosed for PJP were found to be similar to those in literature [9].

The most frequently seen symptoms among HIV (+) patients are dyspnea, cough, wheezing, and expectorating [6]. The application symptoms of our patients followed-up with diagnosis of PCP are in corroboration with the literature. Clinic course was also in corroboration with the literature and 10 of 25 patients were followed up in intensive care unit [6].

In Top 10 diseases causing death in world, TB is in 5th rank, while HIV/AIDS is in 9th rank. HIV positivity was determined in $11 \%$ of 10.4 million newly diagnosed TB and 0.4 million of 1.8 million patients, who died because of TB. In 55\% of TB patients, the HIV test results could be documented. It was determined that $78 \%$ of HIV (+) TB patients were receiving antiretroviral treatment, while TB treatment was initiated for 910.000 newly diagnosed HIV (+) patients [10].

In our country, the rate of HIV test among TB patients is $58.9 \%$ in year 2015 , and there were $45 \mathrm{~TB} / \mathrm{HIV}$ positive $(0.31 \%)$ patients. In Turkey, on the date 03.04.2011, the "Regulation on HIV Diagnostic Test for Tuberculosis Patients" (Nr. 2011/14) was published. For this reason, HIV test can be requested from TB patients without their approval [11].

It is known that TB disease developed 21 times more in HIV (+) patients when compared to HIV (-) ones, and TB patients diagnosed for HIV (+) are strongly recommended to start with early antiretroviral treatment [12].

HIV (+) TB patients are of special importance because of their atypical radiology, different clinic forms, and drug interactions. Different forms of TB were observed in 16 (29.62\%) of 54 patients diagnosed for HIV positivity. From radiological aspect, cavity was seen only in 2 cases. The duration of TB treatment was not changed. But, when used with Protease Inhibitor (PI) and Non-Nucleoside ReverseTranscriptase Inhibitor (NNRTI) drugs, Rifampicin was replaced with Rifabutin, since it was known to decrease the level of these drugs. Since year 2015, antiretroviral treatment is initiated immediately for HIV/ TB patients. According to Guidelines for treatment of drug-susceptible tuberculosis and patient care update 2017, Antiretroviral Treatment (ART) should be started in all TB patients with HIV regardless of their CD4 cell count. TB treatment should be initiated first, followed by ARTas soon as possible within the first 8 week of treatment. HIV (+) patients with profound immunosupression (e.g. CD4counts less than 50 cells $/ \mathrm{mm}^{3}$ ) should receive ART within the first 2 weeks of initiating $\mathrm{TB}$ treatment. In patient with dug susceptible pulmonary $\mathrm{TB}$ who are living with HIV and receiving ART during TB treatment [13].

4 of our patients were serologically diagnosed for CMV pneumonia. For 3 of the patients diagnosed for CMV pneumonia, PCP was suspected but, because of the general condition of patient, no FOB or BAL could be performed and the diagnosis couldn't be confirmed. In literature, both factors were reported to cause pneumonia. For the patients having widespread infiltration in radiology and widespread icy-glass appearance in thoracic computed tomography, we'd like to emphasize that the examinations for revealing both PCP and CMV factors should be performed in case of the presence of HIV/AIDS within the pre-diagnoses $[14,15]$.
HIV-related pulmonary hypertension is a rarely seen (\%0.5) clinical manifestation. Among our patients, the cardiology consultation was requested while examining the etiology of dyspnea and it was observed in colored Doppler. But no analysis regarding HIV-based pulmonary hypertension was performed. We attribute the lack of information to the absence of consensus reports on the subject [16].

\section{Conclusion}

On the contrary with literature, most of our HIV patients were diagnosed for PJP. For the patients that applied with complaints of cough, fever, and dyspnea and then found to have deep hypoxia and extensive infiltration in lung and oral candidiasis in physical examination, hemogram of whom the leucopenia and especially lymphopenia was detected in $P$. jiroveci pneumonia should be considered, thoracic CT - HRCT should be immediately taken and HIV test should be performed.

For the patients diagnosed for tuberculosis, the Elisa test must be requested. In case of HIV $(+)$ condition, the antiretroviral treatment should be immediately initiated, and the treatment should be regulated in the way not degrading the effects of anti-tuberculosis medicaments.

Because the most important one of the organs addressed by HIV infection is the lungs, we should well-know the respiratory system complications, to can identify it immediately and to report it. For this reason, consensus reports on HIV-related diseases are needed in our country.

Unlike TB disease, the approval of patient is needed for testing for HIV. But, the patients, who know that he/she is HIV (+) but do not report it to the physician during anamnesis, poses risk for the healthcare professionals. We believe that the required legal regulations taking healthcare professionals under protection should be prepared.

\section{Funding}

The aim of this study is to draw attention on the importance of lung involvement of HIV infection while examining the cases, which we follow up in University of Health Sciences Sureyyapasa Chest Disease and Chest Surgery Training and Research Hospital. According to our literature research there is no data about this topic in Turkey.

\section{Conflict of interest}

Authors report no conflict of interests related to the current study.

\section{Reference}

1. Dr. Aygen Tumer. AIDS nedir.Dunya ve Turkiyede AIDS. Turk HIV/AIDS Dergisi Hacettepe univ HIV/aids arastırma merkezi

2. Global AIDS UPDATE 2016. http://www.who.int/hiv/pub/arv/global-aids-update2016-pub/en/

3. Mark J Rosen (1996) Pulmonary complications of HIV infections. Clinics in Chest Medicine 17.

4. Beck JM, Rosen MJ, Peavy HH (2001) Pulmonary complications of HIV infections. Report of the fourth NHLBI Workshop. Am J Respir Crit Care Med 164: 2120-2126. [Crossref]

5. Taylor IK, Cooker RJ, Clarke J, Moss FM, Niemann R, et al. (1995) Pulmonary comlications of HIV disease. 10 year retrospective evaluation of yields from broncoalveoler lavage, 1983-93. Thorax 50: 1240-1245. [Crossref]

6. Rosen MJ (1996) Overview of pulmonary complications. Clin Chest Med 17: 621-631.

7. Afessa B, Green B (2000) Bacterial pneumonia in hospitalized patients with HIV infection: the Pulmonary Complications, ICU Support, and Prognostic Factors of Hospitalized Patients with HIV (PIP) Study.. Chest 117: 1017. [Crossref] 
8. Taylor IK1, Coker RJ, Clarke J, Moss FM, Nieman R, et al. (1995) Pulmonary cpmplications of HIV disease: 10 year retrospective evaluation of yields from bronchoalveoler lavage, 1983-93. Thorax 50: 1240-1245. [Crossref]

9. Carolyn M allen, Hamdan H AL-jahdah, Klaus L Irion, Sarah Al Irion, Alaa Gouda, et al. (2010) Imaging lung manifestations of HIV/AIDS. Ann Thorac Med 5: 201-216. [Crossref]

10. Global Tuberculosis report 2016. http://www.who.int/tb/publications/global_report/en/

11. Turkey Verem Savas Raporu, 2014. http://tuberkuloz.thsk.saglık.gov.tr

12. A guide to monitoring and evaluation for collaborative TB/HIV activities, 2015 revision. http://www.who.int/tb/publications/m_and_e_document_page/en/
13. Guidelines for treatment of drug-suscepible and patient care, 2017 update.

14. Gronborg HL, Jespersen S, Honge BL, Jensen-Fangel S, Wejse C (2017) Review of cytomegalovirus coinfection in HIV-infected individuals in Africa. Rev Med Virol 27. [Crossref]

15. Polaczek MM, Zych J, Oniszh K, Szopiński J, Grudny J, et al. (2014) Pneumocystis pneumonia in HIV-infected patients with cytomegalovirus co-infection. Two case reports and a literature review. Pneumonol Alergol Pol 82: 458-466 [Crossref]

16. Speich R, Jenni R, Opravil M, Pfab M, Russ EW (1991) Primary pulmonary hypertension in HIV infection. Chest 100: 1268-1271. [Crossref]

Copyright: $(02017$ Sertçelik L. This is an open-access article distributed under the terms of the Creative Commons Attribution License, which permits unrestricted use, distribution, and reproduction in any medium, provided the original author and source are credited. 